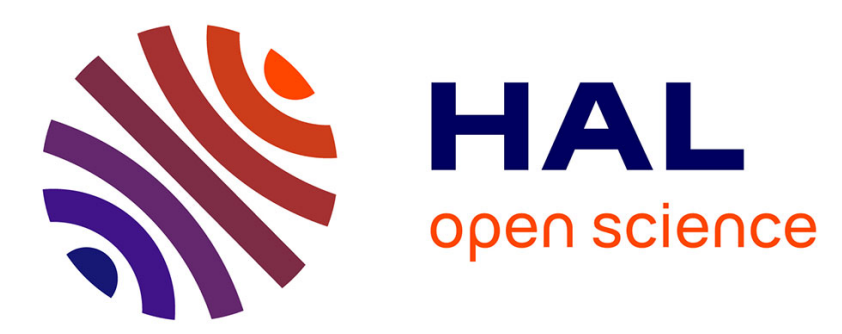

\title{
A posteriori error estimates of the stabilized Crouzeix-Raviart finite element method for the Lamé-Navier equations
}

Emmanuel Creusé, Mohamed Farhloul, Serge Nicaise, Luc Paquet

\section{To cite this version:}

Emmanuel Creusé, Mohamed Farhloul, Serge Nicaise, Luc Paquet. A posteriori error estimates of the stabilized Crouzeix-Raviart finite element method for the Lamé-Navier equations. Far East Journal of Mathematical Sciences, 2015, 96 (2), pp.167-192. 10.17654/FJMSJan2015_167_192 . hal-00777678

\section{HAL Id: hal-00777678 https://hal.science/hal-00777678}

Submitted on 17 Jan 2013

HAL is a multi-disciplinary open access archive for the deposit and dissemination of scientific research documents, whether they are published or not. The documents may come from teaching and research institutions in France or abroad, or from public or private research centers.
L'archive ouverte pluridisciplinaire HAL, est destinée au dépôt et à la diffusion de documents scientifiques de niveau recherche, publiés ou non, émanant des établissements d'enseignement et de recherche français ou étrangers, des laboratoires publics ou privés. 


\title{
A POSTERIORI ERROR ESTIMATES OF THE STABILIZED CROUZEIX-RAVIART FINITE ELEMENT METHOD FOR THE LAMÉ-NAVIER EQUATIONS.
}

\author{
E. CREUSÉ * ${ }^{*}$ M. FARHLOUL ${ }^{\dagger}$, S. NICAISE $\ddagger$, AND L. PAQUET $\ddagger$
}

\begin{abstract}
We obtain a posteriori error estimates for a variant of (non-locking) stabilized nonconforming methods based on the Crouzeix-Raviart element introduced by P. Hansbo and M. G. Larson in [M2AN 37 (2003) 63-72]. We derive upper and lower a posteriori error bounds which are robust with respect to the nearly incompressible materials.
\end{abstract}

Key words. Crouzeix-Raviart element, nonconforming method, stabilized method, nonlocking, a posteriori error estimates.

AMS subject classifications. $65 \mathrm{~N} 30,65 \mathrm{~N} 15,65 \mathrm{~N} 50$

1. Introduction. The finite element methods are widely used for the numerical approximation of many problems occurring in engineering applications, like the Laplace equation, the Lamé system, etc.... (see $[10,18]$ ). In practice, adaptive techniques based on a posteriori error estimators have become indispensable tools for such methods. Hence there now exists a large number of publications devoted to the analysis of some finite element approximations of problems from solid mechanics and obtaining locally defined a posteriori error estimates. We refer to the monographs $[2,7,29]$ for a good overview on this topic.

For the elasticity system in the primal variables, several different approaches have been developed: Residual type error estimators [4, 5, 17, 30, 31, 11], methods based on the resolution of local subproblems by using higher order elements $[4,6,8]$, averaging techniques (the so-called Zienkiewicz-Zhu estimators) $[1,2,32,33]$ and finally estimators based on equilibrated fluxes [3, 12, 23, 24, 25, 27]. For methods based on dual variables, like mixed methods, we refer to [13, 14, 16, 9, 22]; note that such methods are usually locking free and therefore the obtained estimators are usually locking free.

Here we analyze two displacement methods based on the primal variables variant of the ones introduced by P. Hansbo and M.G. Larson in [20] which are nonconforming methods based on the Crouzeix-Raviart finite element. These methods are locking free and very cheap. In [20], the authors derive a priori optimal error estimates uniform in the Lamé parameter $\lambda$ (see Theorem 3.1 of [20]). In this paper, we propose an a posteriori error analysis of these methods. Our analysis enters in the family of estimators of residual type since our error indicator is based on residuals on each triangle and jumps across the inter-element boundaries. We prove reliability of the indicator uniformly in $\lambda$ (and $h$ ), in particular avoiding locking phenomena. The proof is based on a discrete divergence stability result (see [11] for another scheme), the use of an appropriate decomposition of the error into continuous and discontinuous parts

\footnotetext{
${ }^{*}$ Université Lille 1 - Laboratoire Paul Painlevé and E.P.I. SIMPAF - INRIA Lille Nord Europe, Cité scientifique, 59655 Villeneuve d'Ascq Cedex, France (creuse@math.univ-lille1.fr).

$\dagger$ Université de Moncton, Département de Mathématiques et de Statistique, Moncton, N.B., E1A 3E9, Canada (mohamed.farhloul@umoncton.ca).

¥Université de Valenciennes et du Hainaut Cambrésis, LAMAV, Institut des Sciences et Techniques de Valenciennes, F-59313 - Valenciennes Cedex 9 France ( $\{$ Serge.Nicaise, Luc.Paquet\}@univ-valenciennes.fr).
} 
[21]. Local efficiency of our indicator follows by using classical inverse estimates, the use of a strengthened norm allows to obtain its robustness.

Note that our methods enter in a similar framework than the ones in [17, 31, 11]. But our upper bound cannot be deduced from [17] because the assumption (2.12) from that paper is not satisfied by our scheme. On the other hand, the schemes in $[17,31,11]$ are different from our proposed schemes, nevertheless the proof of the upper bound uses similar arguments than in [11].

The outline of the paper is as follows: We recall in Section 2 the boundary value problem and its numerical approximation. Section 3 is devoted to the proof of the discrete divergence stability result. The proof of the upper error bound is made in section 4; and the lower bound is obtained in section 5 . Finally some numerical tests that confirm our theoretical results are presented in the last section.

Let us finish this introduction with some notations used in the remainder of the paper: On $D$, the $L^{2}(D)$-norm will be denoted by $\|\cdot\|_{D}$. The usual norm and seminorm of $H^{s}(D)(s \geq 0)$ are denoted by $\|\cdot\|_{s, D}$ and $|\cdot|_{s, D}$, respectively. In the case $D=\Omega$, the index $\Omega$ will be omitted. Similar $H(\operatorname{div}, \Omega)$ is defined by

$$
H(\operatorname{div}, \Omega)=\left\{v \in L^{2}(\Omega)^{2}: \operatorname{div} v \in L^{2}(\Omega)\right\},
$$

equipped with its natural norm. Finally, the notation $a \lesssim b$ means the existence of a positive constant $C$, which is independent of the mesh size, of the considered quantities $a$ and $b$ and of the Lamé coefficient $\lambda$ such that $a \leq C b$. In other words, the constant only depends on the aspect ratio of the mesh, on the domain $\Omega$ and on the shear modulus $\mu$, but not on Lamé coefficient $\lambda$ (see below).

\section{A discontinuous Galerkin method for the elasticity system.}

2.1. The boundary value problem of elasticity. Let $\Omega$ be a plane domain with a polygonal boundary. More precisely, we assume that $\Omega$ is a simply connected domain and that its boundary $\Gamma$ is the union of a finite number of linear segments $\bar{\Gamma}_{j}$, $1 \leq j \leq n_{e}$ ( $\Gamma_{j}$ is assumed to be an open segment). In this domain, we consider an elastic isotropic material. Let $u=\left(u_{1}, u_{2}\right)$ be the displacement field and $f=\left(f_{1}, f_{2}\right) \in$ $\left[L^{2}(\Omega)\right]^{2}$ the body force by unit of mass. For simplicity, we consider in $\Omega$ the pure homogeneous displacement problem. Thus the displacement field $u=\left(u_{1}, u_{2}\right)$ satisfies the following equations and boundary condition:

$$
\left\{\begin{aligned}
&-\operatorname{div} \sigma(u)=f \quad \text { in } \Omega, \\
& u=0 \text { on } \Gamma
\end{aligned}\right.
$$

where the stress tensor is defined by

$$
\sigma(u):=2 \mu \epsilon(u)+\lambda \operatorname{tr} \epsilon(u) I .
$$

The positive constants $\mu$ and $\lambda$ are called the Lamé coefficients where $\mu \equiv G$ is the shear modulus ([28], p. 207) ([19], pp. 72-75, 57-58). We assume that [10]

$$
(\lambda, \mu) \in\left[\lambda_{0},+\infty\left[\times\left[\mu_{1}, \mu_{2}\right]\right.\right.
$$

where

$$
0<\mu_{1}<\mu_{2} \text { and } \quad \lambda_{0}>0
$$


As usual, $\epsilon(u)$ denotes the linearized strain tensor, i.e., $\epsilon(u):=\frac{1}{2}\left(\nabla u+(\nabla u)^{T}\right)$ and $I$ the identity tensor. For any tensor $\tau$ (of order 2), the trace of $\tau$ is $\operatorname{tr} \tau:=\tau_{11}+\tau_{22}$, and

$$
\operatorname{div} \tau:=\left(\frac{\partial \tau_{11}}{\partial x_{1}}+\frac{\partial \tau_{12}}{\partial x_{2}}, \frac{\partial \tau_{21}}{\partial x_{1}}+\frac{\partial \tau_{22}}{\partial x_{2}}\right)
$$

(in an orthogonal cartesian frame). The classical variational formulation of the boundary value problem $(2.1)$ is the following: find $u \in\left[H_{0}^{1}(\Omega)\right]^{2}$ such that

$$
\int_{\Omega} \sigma(u): \epsilon(v) d x=\int_{\Omega} f \cdot v d x, \forall v \in\left[H_{0}^{1}(\Omega)\right]^{2},
$$

where $\sigma: \epsilon$ denotes the contraction of the two tensors, i.e.,

$$
\sigma: \epsilon=\sum_{i, j=1}^{2} \sigma_{i j} \epsilon_{i j} .
$$

The existence and uniqueness of the solution in (2.4), for a given $f \in\left[H^{-1}(\Omega)\right]^{2}$, follows from the first Korn inequality (cf. [10], p.286). In the following, we suppose that $f \in\left[L^{2}(\Omega)\right]^{2}$.

2.2. The discontinuous Galerkin methods. In order to formulate the discrete problems introduced by P. Hansbo and M.G. Larson in [20], we first recall some notation.

Let $\left(\mathcal{T}_{h}\right)_{h>0}$, be a regular family of triangulations. Let us fix a triangulation $\mathcal{T}_{h} \in\left(\mathcal{T}_{h}\right)_{h>0}$ and let $\mathcal{E}_{h}$ denote the set of all edges of the triangulation $\mathcal{T}_{h}$. We split $\mathcal{E}_{h}$ into two disjoint subsets: $\mathcal{E}_{h}^{I}$ the subset of edges contained in $\Omega$ and $\mathcal{E}_{h}^{\partial}$ the subset of edges contained in the boundary of $\Omega$. Further, with each edge $E$, we associate a fixed unit normal $n_{E}$ such that for edges contained in $\partial \Omega, n_{E}$ is the exterior unit normal; we further fix a unit tangent $t_{E}$ so that $\left\{n_{E}, t_{E}\right\}$ forms an orthonormal basis of $\mathbb{R}^{2}$. For any edge $E$ and any triangle $T$, we denote by $|E|$ the length of $E$ and $|T|$ the area of $T$.

Let

$$
D F\left(\mathcal{T}_{h}\right):=\left\{v_{h} \in\left[L^{2}(\Omega)\right]^{2} ; \forall T \in \mathcal{T}_{h}: v_{h \mid T} \in\left[P_{1}(T)\right]^{2}\right\}
$$

be the space of piecewise linear discontinuous functions (as usual $P_{1}(T)$ denotes the space of polynomials of degree less than or equal to 1 on $T$ ).

For a function $v_{h} \in D F\left(\mathcal{T}_{h}\right)$ we denote its jump at an edge $E$ by $\left[\left[v_{h}\right]\right]_{E}=v_{h}^{+}-v_{h}^{-}$ for $E \in \mathcal{E}_{h}^{I}$ and $\left[\left[v_{h}\right]\right]_{E}=v_{h}^{+}$for $E \in \mathcal{E}_{h}^{\partial}$, and the average $\left\langle v_{h}\right\rangle_{E}=\left(v_{h}^{+}+v_{h}^{-}\right) / 2$ for $E \in \mathcal{E}_{h}^{I}$ and $\left\langle v_{h}\right\rangle_{E}=v_{h}^{+}$for $E \in \mathcal{E}_{h}^{\partial}$, where $v_{h}^{ \pm}(x)=\lim _{h \rightarrow 0^{+}} v_{h}\left(x \mp h n_{E}\right)$ with $x \in E$. In the same spirit, we define its broken strain tensor $\epsilon_{h}\left(v_{h}\right)$ by

$$
\epsilon_{h}\left(v_{h}\right)_{T}=\epsilon\left(v_{h \mid T}\right) \quad \forall T \in \mathcal{T}_{h},
$$

its broken stress tensor $\sigma_{h}\left(v_{h}\right)$ and broken divergence $\operatorname{div}_{h} v_{h}$ is defined similarly.

We further introduce the Crouzeix-Raviart space of vector fields $C R\left(\mathcal{T}_{h}\right)$ :

$$
C R\left(\mathcal{T}_{h}\right):=\left\{v_{h} \in\left[L^{2}(\Omega)\right]^{2} ; \forall T \in \mathcal{T}_{h}: v_{h \mid T} \in\left[P_{1}(T)\right]^{2},\left[\left[P_{0} v_{h}\right]\right]_{E}=0, \forall E \in \mathcal{E}_{h}\right\}
$$


where for any edge $E$ and any $w \in L^{2}(E)$

$$
P_{0} w:=\frac{1}{|E|} \int_{E} w d s
$$

and therefore

$$
\left[\left[P_{0} v_{h}\right]\right]_{E}:=P_{0}\left[\left[v_{h}\right]\right]_{E}=\frac{1}{|E|} \int_{E} v_{h}^{+} d s-\frac{1}{|E|} \int_{E} v_{h}^{-} d s .
$$

The first discontinuous Galerkin method is a variant of the one introduced in [20] and reads as follows: find $u_{h} \in D F\left(\mathcal{T}_{h}\right)$ such that

$$
a_{h}\left(u_{h}, v_{h}\right)=\left(f, v_{h}\right), \forall v_{h} \in D F\left(\mathcal{T}_{h}\right) .
$$

The bilinear form is here defined by

$$
\begin{array}{r}
\left.a_{h}\left(u_{h}, v_{h}\right):=a_{0 h}\left(u_{h}, v_{h}\right)+(2 \mu+\lambda) \lambda \gamma_{0} \sum_{E \in \mathcal{E}_{h}} \frac{1}{h_{E}}\left(\left[\left[P_{0} u_{h} \cdot n_{E}\right]\right],\left[\left[P_{0} v_{h} \cdot n_{E}\right]\right]\right)_{E} 2.7\right) \\
+2 \mu \gamma_{0}^{\prime} \sum_{E \in \mathcal{E}_{h}} \frac{1}{h_{E}}\left(\left[\left[P_{0} u_{h} \cdot t_{E}\right]\right],\left[\left[P_{0} v_{h} \cdot t_{E}\right]\right]\right)_{E} \\
\quad-\sum_{E \in \mathcal{E}_{h}}\left(\left(\left\langle\sigma_{h}\left(u_{h}\right)\right\rangle_{E} n_{E},\left[\left[v_{h}\right]\right]_{E}\right)_{E}+\left(\left\langle\sigma_{h}\left(v_{h}\right)\right\rangle_{E} n_{E},\left[\left[u_{h}\right]\right]_{E}\right)_{E}\right), \\
a_{0 h}\left(u_{h}, v_{h}\right):=\sum_{T \in \mathcal{T}_{h}}\left(\sigma\left(u_{h}\right), \epsilon\left(v_{h}\right)\right)_{T}+2 \mu \gamma_{1} \sum_{E \in \mathcal{E}_{h}} \frac{1}{h_{E}}\left(\left[\left[u_{h}\right]\right],\left[\left[v_{h}\right]\right]\right)_{E}
\end{array}
$$

while the linear form is defined by

$$
\left(f, v_{h}\right)=\int_{\Omega} f \cdot v_{h} d x .
$$

Here the parameters $\gamma_{0}, \gamma_{0}^{\prime}$ and $\gamma_{1}$ are the stabilizing parameters fixed below.

Here and below $\left(\sigma\left(u_{h}\right), \epsilon\left(v_{h}\right)\right)_{T}$ means the $L^{2}$-inner product $\int_{T} \sigma\left(u_{h}\right): \epsilon\left(v_{h}\right) d x$. Similarly for vector valued functions $v, w \in L^{2}(E)^{2},(v, w)_{E}$ means the $L^{2}$-inner product $\int_{E} v \cdot w d s$.

Note that our bilinear form $a_{h}$ differs from the one in [20] by the second and third terms of the right-hand side of (2.7), indeed in that paper these two terms are replaced by

$$
(2 \mu+\lambda) \gamma_{0} \sum_{E \in \mathcal{E}_{h}} \frac{1}{h_{E}}\left(\left[\left[P_{0} u_{h}\right]\right],\left[\left[P_{0} v_{h}\right]\right]\right)_{E} \cdot
$$

This modification is made to have a discrete stability result and hence a robust a posteriori estimate that cannot be obtained if we keep the original term from [20]. Note further that the bilinear form $a_{h}$ is similar to the one in [11] except that the second and third terms are replaced by

$$
\lambda^{2} \gamma_{0} \sum_{E \in \mathcal{E}_{h}} \frac{1}{h_{E}}\left(\left[\left[u_{h} \cdot n_{E}\right]\right],\left[\left[v_{h} \cdot n_{E}\right]\right]\right)_{E} .
$$


As a consequence the well-posedness of the corresponding problem in [11] is obtained for $\gamma_{1}$ and $\gamma_{0}$ large enough (independently of $\lambda$ ), while our problem is well-posed only for $\gamma_{0}$ and $\gamma_{0}^{\prime}$ large enough (independently of $\lambda$ ). Our approach allows then to use a penalization parameter $\gamma_{1}$ as small as we want (but bounded away from zero). This difference also implies the use of a different error norm.

The well-posedness of problem (2.6) follows from the strong coerciveness of $a_{h}$ in $D F\left(\mathcal{T}_{h}\right)$, that we now show (see Proposition 2.2 of [20] or Proposition 2.2 of [11])

Proposition 2.1. There exist three positive constants $\gamma_{\min }, \gamma_{\text {min }}^{\prime}$ and $C$ independent of $\lambda, \mu$ and the mesh size $h$ such that for all $\gamma_{0}>\gamma_{\text {min }}$ and all $\gamma_{0}^{\prime}>\gamma_{\text {min }}^{\prime}$ and any $v_{h} \in D F\left(\mathcal{T}_{h}\right)$, we have

$$
\begin{aligned}
a_{h}\left(v_{h}, v_{h}\right) & \geq C\left(2 \mu\left\|\epsilon_{h}\left(v_{h}\right)\right\|^{2}+\lambda\left\|\operatorname{div}_{h} v_{h}\right\|^{2}\right. \\
& +(2 \mu+\lambda) \lambda \sum_{E \in \mathcal{E}_{h}} \frac{1}{h_{E}}\left\|\left[\left[P_{0} v_{h} \cdot n_{E}\right]\right]\right\|_{E}^{2} \\
& \left.+2 \mu \sum_{E \in \mathcal{E}_{h}} \frac{1}{h_{E}}\left\|\left[\left[P_{0} v_{h} \cdot t_{E}\right]\right]\right\|_{E}^{2}+2 \mu \gamma_{1} \sum_{E \in \mathcal{E}_{h}} \frac{1}{h_{E}}\left\|\left[\left[v_{h}\right]\right]\right\|_{E}^{2}\right)
\end{aligned}
$$

Proof. First for an arbitrary edge $E$, we have

$$
\left(\left\langle\sigma_{h}\left(v_{h}\right)\right\rangle_{E} n_{E},\left[\left[v_{h}\right]\right]_{E}\right)_{E}=2 \mu\left(\left\langle\epsilon\left(v_{h}\right)\right\rangle_{E} n_{E},\left[\left[v_{h}\right]\right]_{E}\right)_{E}+\lambda\left(\left[\left[\operatorname{div}_{h} v_{h}\right]\right],\left[\left[v_{h} \cdot n_{E}\right]\right]_{E}\right)_{E}
$$

and since $\epsilon_{h}\left(v_{h}\right)$ and $\operatorname{div}_{h} v_{h}$ are piecewise constant, we get

$$
\left(\left\langle\sigma_{h}\left(v_{h}\right)\right\rangle_{E} n_{E},\left[\left[v_{h}\right]\right]_{E}\right)_{E}=2 \mu\left(\left\langle\epsilon\left(v_{h}\right)\right\rangle_{E} n_{E},\left[\left[P_{0} v_{h}\right]\right]_{E}\right)_{E}+\lambda\left(\left[\left[\operatorname{div}_{h} v_{h}\right]\right],\left[\left[P_{0} v_{h} \cdot n_{E}\right]\right]_{E}\right){ }_{E}
$$

By Cauchy-Schwarz's and Young's inequalities we obtain

$$
\begin{aligned}
\left|\left(\left\langle\sigma_{h}\left(v_{h}\right)\right\rangle_{E} n_{E},\left[\left[v_{h}\right]\right]_{E}\right)_{E}\right| & \leq \frac{\mu}{\delta^{\prime}} h_{E}\left\|\left\langle\epsilon\left(v_{h}\right)\right\rangle_{E}\right\|_{E}^{2} \\
& +\mu \delta^{\prime} h_{E}^{-1}\left\|\left[\left[P_{0} v_{h}\right]\right]_{E}\right\|_{E}^{2} \\
& +\frac{\lambda}{2(2 \mu+\lambda) \delta} h_{E}\left\|\left[\left[\operatorname{div}_{h} v_{h}\right]\right]_{E}\right\|_{E}^{2} \\
& +\frac{\lambda(2 \mu+\lambda) \delta}{2} h_{E}^{-1}\left\|\left[\left[P_{0} v_{h} \cdot n_{E}\right]\right]_{E}\right\|_{E}^{2}
\end{aligned}
$$

for any $\delta, \delta^{\prime}>0$. Using the regularity assumption of the mesh, there exists a positive constant $C_{t r}$ such that

$$
\begin{array}{r}
h_{E}\left\|\left[\left[\operatorname{div}_{h} v_{h}\right]\right]_{E}\right\|_{E}^{2} \leq C_{t r}\left\|\operatorname{div}_{h} v_{h}\right\|_{\omega_{E}}^{2}, \\
h_{E}\left\|\left\langle\epsilon\left(v_{h}\right)\right\rangle_{E}\right\|_{E}^{2} \leq C_{t r}\left\|\epsilon_{h}\left(v_{h}\right)\right\|_{\omega_{E}}^{2},
\end{array}
$$

where, as usual, $\omega_{E}$ is the patch associated with $E$, i.e., $\omega_{E}=T_{E}^{+} \cup T_{E}^{-}$, when $E$ is the common edge between $T_{E}^{+}$and $T_{E}^{-}$. These estimates in the previous one lead to 


$$
\begin{aligned}
\left|\sum_{E \in \mathcal{E}_{h}}\left(\left\langle\sigma_{h}\left(v_{h}\right)\right\rangle_{E} n_{E},\left[\left[v_{h}\right]\right]_{E}\right)_{E}\right| & \leq \frac{2 \mu C_{t r}}{\delta^{\prime}}\left\|\epsilon_{h}\left(v_{h}\right)\right\|^{2} \\
& +\mu \delta^{\prime} \sum_{E \in \mathcal{E}_{h}} h_{E}^{-1}\left\|\left[\left[P_{0} v_{h}\right]\right]_{E}\right\|_{E}^{2} \\
& +\frac{\lambda C_{t r}}{(2 \mu+\lambda) \delta}\left\|\operatorname{div}_{h} v_{h}\right\|^{2} \\
& +\frac{\lambda(2 \mu+\lambda) \delta}{2} \sum_{E \in \mathcal{E}_{h}} h_{E}^{-1}\left\|\left[\left[P_{0} v_{h} \cdot n_{E}\right]\right]_{E}\right\|_{E}^{2} .
\end{aligned}
$$

By this estimate and the definition of $a_{h}$, we deduce that

$$
\begin{aligned}
a_{h}\left(v_{h}, v_{h}\right) & \geq 2 \mu\left(1-\frac{2 C_{t r}}{\delta^{\prime}}\right)\left\|\epsilon_{h}\left(v_{h}\right)\right\|^{2}+\lambda\left(1-\frac{2 C_{t r}}{(2 \mu+\lambda) \delta}\right)\left\|\operatorname{div}_{h} v_{h}\right\|^{2} \\
& +2 \mu \gamma_{1} \sum_{E \in \mathcal{E}_{h}} \frac{1}{h_{E}}\left\|\left[\left[v_{h}\right]\right]_{E}\right\|_{E}^{2} \\
& +(2 \mu+\lambda) \lambda\left(\gamma_{0}-\delta-\frac{2 \mu \delta^{\prime}}{(2 \mu+\lambda) \lambda}\right) \sum_{E \in \mathcal{E}_{h}} \frac{1}{h_{E}}\left\|\left[\left[P_{0} v_{h} \cdot n_{E}\right]\right]\right\|_{E}^{2} \\
& +2 \mu\left(\gamma_{0}^{\prime}-\delta^{\prime}\right) \sum_{E \in \mathcal{E}_{h}} \frac{1}{h_{E}}\left\|\left[\left[P_{0} v_{h} \cdot t_{E}\right]\right]_{E}\right\|_{E}^{2} .
\end{aligned}
$$

Now we choose $\delta$ and $\delta^{\prime}$ such that

$$
\left(1-\frac{2 C_{t r}}{\delta^{\prime}}\right)=\left(1-\frac{2 C_{t r}}{(2 \mu+\lambda) \delta}\right)=\frac{1}{2},
$$

or equivalently

$$
\delta^{\prime}=4 C_{t r} \text { and } \delta=\frac{4 C_{t r}}{(2 \mu+\lambda)} .
$$

With this choice we then have

$$
\begin{aligned}
a_{h}\left(v_{h}, v_{h}\right) & \geq \mu\left\|\epsilon_{h}\left(v_{h}\right)\right\|^{2}+\frac{\lambda}{2}\left\|\operatorname{div}_{h} v_{h}\right\|^{2} \\
& +2 \mu \gamma_{1} \sum_{E \in \mathcal{E}_{h}} \frac{1}{h_{E}}\left\|\left[\left[v_{h}\right]\right]_{E}\right\|_{E}^{2} \\
& +(2 \mu+\lambda) \lambda\left(\gamma_{0}-\frac{4 C_{t r}}{(2 \mu+\lambda)}-\frac{8 \mu C_{t r}}{(2 \mu+\lambda) \lambda}\right) \sum_{E \in \mathcal{E}_{h}} \frac{1}{h_{E}}\left\|\left[\left[P_{0} v_{h} \cdot n_{E}\right]\right]\right\|_{E}^{2} \\
& +2 \mu\left(\gamma_{0}^{\prime}-4 C_{t r}\right) \sum_{E \in \mathcal{E}_{h}} \frac{1}{h_{E}}\left\|\left[\left[P_{0} v_{h} \cdot t_{E}\right]\right]_{E}\right\|_{E}^{2} .
\end{aligned}
$$

The conclusion follows with the choice

$$
\gamma_{\text {min }}=\max \left\{1, \frac{4 C_{t r}}{(2 \mu+\lambda)}+\frac{8 \mu C_{t r}}{(2 \mu+\lambda) \lambda}\right\} \text { and } \gamma_{\text {min }}^{\prime}=4 C_{t r} .
$$


By the previous result, we deduce that for $\gamma_{0}>\gamma_{\min }$ and $\gamma_{0}^{\prime}>\gamma_{\min }^{\prime}$, problem (2.6) is well posed.

As shown in Proposition 2.1 of [20] the method is consistent, namely

$$
a_{h}\left(u-u_{h}, v_{h}\right)=0, \forall v_{h} \in D F\left(\mathcal{T}_{h}\right) .
$$

Restricting the discontinuous Galerkin method to the space of Crouzeix-Raviart functions, we obtain the following simplified scheme: find $u_{0 h} \in C R\left(\mathcal{T}_{h}\right)$ such that

$$
a_{0 h}\left(u_{0 h}, v_{h}\right)=\left(f, v_{h}\right), \forall v_{h} \in C R\left(\mathcal{T}_{h}\right) .
$$

As before this second method is consistent:

$$
a_{0 h}\left(u-u_{0 h}, v_{h}\right)=0, \forall v_{h} \in C R\left(\mathcal{T}_{h}\right) .
$$

Observe that Theorem 2.3 of [26] guarantees that the displacement vector field $u \in\left[H^{3 / 2+\epsilon}(\Omega)\right]^{2}$ for some $\left.\epsilon \in\right] 0, \frac{1}{2}\left[\right.$. Consequently $\sigma(u) \cdot n_{E}$ has sense on each edge $E \in \mathcal{E}_{h}$ as an element of $\left[H^{\epsilon}(E)\right]^{2} \subset\left[L^{2}(E)\right]^{2}$ and Green's formula can be applied element by element. Hence the consistency always holds.

We finally refer to $[20]$ for robust a priori error estimates.

Our goal is to bound the "triple norm" $\||e|\|:=\sqrt{a_{0 h}(e, e)}$ of the error

$$
e:=u-u_{h} .
$$

But in order to get a robust estimate we even need to use the parameter depending norm:

$$
\|\| \mid\left\|_{\lambda}^{2}=\right\|\|e\|\left\|^{2}+\lambda^{2}\right\| \Pi_{0} d i v_{h} e\left\|^{2}+\lambda^{2} \sum_{E \in \mathcal{E}_{h}} \frac{1}{h_{E}}\right\|\left[\left[P_{0} e \cdot n_{E}\right]\right] \|_{E}^{2},
$$

where div $_{h} e$ means the broken divergence and $\Pi_{0}$ is the $L^{2}$-projection on $L_{0}^{2}(\Omega)^{2}$ defined by

$$
\Pi_{0} f=f-\frac{1}{|\Omega|} \int_{\Omega} f(x) d x, \forall f \in L^{2}(\Omega)^{2} .
$$

Before going on notice that if $u_{0 h} \in C R\left(\mathcal{T}_{h}\right)$, then

$$
\int_{\Omega} \operatorname{div}_{h} u_{0 h} d x=0
$$

3. Discrete stability. The stability of the continuous problem (2.4) was proved in Proposition 3.2 of [11] and means that there exists a positive constant $c$ independent of $\lambda$ such that

$$
\lambda\|\operatorname{div} u\| \leq c\|f\| .
$$

A discrete version of this estimate was also proved in Corollary 3.7 of [11] for their own scheme. Using similar arguments we also obtain a discrete stability.

Proposition 3.1. If $u_{h} \in D F\left(\mathcal{T}_{h}\right)$ is the solution of (2.6), then

$$
\left\|\left|u_{h}\right|\right\|_{\lambda} \lesssim\|f\| .
$$


Proof. We first use Lemma 3.5 of [11] that yields $w \in D F\left(\mathcal{T}_{h}\right) \cap H(\operatorname{div}, \Omega)$ such that

$$
\begin{array}{r}
\operatorname{div} w=-\lambda \Pi_{0}\left(\operatorname{div}_{h} u_{h}\right), \\
\left\|\nabla_{h} w\right\| \lesssim \lambda\left\|\Pi_{0}\left(\operatorname{div}_{h} u_{h}\right)\right\|, \\
\sum_{E \in \mathcal{E}_{h}} \frac{1}{h_{E}}\|[[w]]\|_{E}^{2} \lesssim \lambda^{2}\left\|\Pi_{0}\left(\operatorname{div}_{h} u_{h}\right)\right\|^{2} .
\end{array}
$$

With such a choice we notice that in $a_{h}\left(u_{h}, w\right)$ the term

$$
(2 \mu+\lambda) \lambda \gamma_{0} \sum_{E \in \mathcal{E}_{h}} \frac{1}{h_{E}}\left(\left[\left[P_{0} u_{h} \cdot n_{E}\right]\right],\left[\left[P_{0} w \cdot n_{E}\right]\right]\right)_{E},
$$

is zero and therefore the arguments of Proposition 3.6 of [11] can be applied.

4. Robust upper bound of the error. For all $T \in \mathcal{T}_{h}$, the local estimator $\eta_{T}$ is defined by

$\eta_{T}^{2}=h_{T}^{2}\left\|f_{T}\right\|_{T}^{2}+\sum_{E \in \mathcal{E}_{h}: E \subset T} h_{E}\left\|\left[\left[\sigma\left(u_{h}\right) \cdot n_{E}\right]\right]_{E}\right\|_{E}^{2}+\sum_{E \in \mathcal{E}_{h}: E \subset T} \frac{1}{h_{E}}\left(\left\|\left[\left[u_{h}\right]\right]\right\|_{E}^{2}+\lambda^{2}\left\|\left[\left[P_{0} u_{h} \cdot n_{E}\right]\right]\right\|_{E}^{2}\right)$,

where $f_{T}=\frac{1}{|T|} \int_{T} f(x) d x$. The global estimator is given by

$$
\eta^{2}=\sum_{T \in \mathcal{T}_{h}} \eta_{T}^{2}
$$

Finally let

$$
\operatorname{osc}^{2}(f)=\sum_{T \in \mathcal{T}_{h}} h_{T}^{2}\left\|f-f_{T}\right\|_{T}^{2}
$$

be the oscillating term.

Now we are able to state our locking-free a posteriori error estimate:

THEOREM 4.1. With the previous notation, we have the following a posteriori estimate:

$$
\|||\|_{\lambda} \lesssim \eta+o s c(f)
$$

Proof. We proceed as in the proof of Theorem 4.2 of [11]. Namely we take $\omega \in H_{0}^{1}(\Omega)^{2}$ such that

$$
\begin{array}{r}
\operatorname{div} \omega=\lambda \Pi_{0}\left(\operatorname{div}_{h} e\right), \\
\|\omega\|_{1, \Omega} \lesssim \lambda\left\|\Pi_{0}\left(\operatorname{div}_{h} u_{h}\right)\right\|,
\end{array}
$$

and such that there exist $\beta>0$ and $\kappa>0$ for which

$$
4 \mu\left\|\epsilon\left(e^{c}\right)\right\|^{2}+\lambda^{2}\left\|\Pi_{0}\left(\operatorname{div}_{h} e\right)\right\|^{2} \leq \kappa\left(2 \mu\left(\epsilon\left(e^{c}\right), \epsilon\left(e^{c}+\beta \omega\right)\right)+\beta \lambda\left(\operatorname{div}_{h} e, \operatorname{div} \omega\right)\right),
$$

where, as usual (see for instance $[21,11]$ ), $e^{c}$ is the continuous part of the error, namely

$$
e^{c}=u-u_{h}^{c}
$$


and

$$
u_{h}=u_{h}^{c}+u_{h}^{\perp},
$$

with $u_{h}^{c} \in V_{h}^{c}:=D F\left(\mathcal{T}_{h}\right) \cap H_{0}^{1}(\Omega)^{2}$ and $u_{h}^{\perp} \in D F\left(\mathcal{T}_{h}\right)^{\perp}$, where $D F\left(\mathcal{T}_{h}\right)^{\perp}$ is an appropriate orthogonal complement of $V_{h}^{c}$ into $D F\left(\mathcal{T}_{h}\right)$.

In a first step using this splitting and the standard estimate

$$
\left\|\nabla_{h} u_{h}^{\perp}\right\|^{2} \lesssim \sum_{E \in \mathcal{E}_{h}} \frac{1}{h_{E}}\left\|\left[\left[u_{h}\right]\right]\right\|_{E}^{2}
$$

we have (compare with the estimate (29) from [11])

$$
\begin{aligned}
\|\| \|_{\lambda}^{2} & \lesssim\left\|\epsilon\left(e^{c}\right)\right\|^{2}+\lambda^{2}\left\|\Pi_{0}\left(\operatorname{div}_{h} e\right)\right\|^{2} \\
& +\sum_{E \in \mathcal{E}_{h}} \frac{1}{h_{E}}\left(\left\|\left[\left[u_{h}\right]\right]\right\|_{E}^{2}+\lambda^{2}\left\|\left[\left[P_{0} u_{h} \cdot n_{E}\right]\right]\right\|_{E}^{2}\right) .
\end{aligned}
$$

Hence it remains to estimate the first two terms of this right-hand side. For that purpose, we use (4.2) and transform its right-hand side as in [11] to get

$$
\begin{array}{r}
4 \mu\left\|\epsilon\left(e^{c}\right)\right\|^{2}+\lambda^{2}\left\|\Pi_{0}\left(\operatorname{div}_{h} e\right)\right\|^{2} \lesssim(\sigma(u), \epsilon(w))-\left(\sigma_{h}\left(u_{h}\right), \epsilon(w)\right) \\
+2 \mu\left(\epsilon\left(u_{h}^{\perp}\right), \epsilon(w)\right)-\lambda\left(\operatorname{div}_{h} e, \operatorname{div} e^{c}\right),
\end{array}
$$

where $w=e^{c}+\beta \omega$ that belongs to $H_{0}^{1}(\Omega)^{2}$ and satisfies (see [11, p. 1550])

$$
\|w\|_{1, \Omega} \lesssim\|\| \|_{\lambda}
$$

For the two first terms of this right-hand side we use the continuous and discrete formulation to get successively (with $w_{h}=I_{C l}(w)$ the Clément interpolant of $w$ )

$$
\begin{aligned}
(\sigma(u), \epsilon(w))-\left(\sigma_{h}\left(u_{h}\right), \epsilon(w)\right) & =\left(\sigma(u), \epsilon\left(w-w_{h}\right)\right)-\left(\sigma_{h}\left(u_{h}\right), \epsilon\left(w-w_{h}\right)\right) \\
& +\left(\sigma(u), \epsilon\left(w_{h}\right)\right)-\left(\sigma_{h}\left(u_{h}\right), \epsilon\left(w_{h}\right)\right) \\
& =\left(\sigma(u), \epsilon\left(w-w_{h}\right)\right)-\left(\sigma_{h}\left(u_{h}\right), \epsilon\left(w-w_{h}\right)\right) \\
& -\sum_{E \in \mathcal{E}_{h}}\left(\left\langle\sigma\left(w_{h}\right)\right\rangle_{E} n_{E},\left[\left[u_{h}\right]\right]_{E}\right)_{E} .
\end{aligned}
$$

Hence using again the continuous formulation and piecewiese Green's formula, we arrive at

$$
\begin{aligned}
(\sigma(u), \epsilon(w))-\left(\sigma_{h}\left(u_{h}\right), \epsilon(w)\right) & =\left(f, w-w_{h}\right) \\
& -\sum_{E \in \mathcal{E}_{h}}\left(\left(\left[\left[\sigma_{h}\left(u_{h}\right) \cdot n_{E}\right]\right], w_{h}\right)_{E}+\left(\left\langle\sigma\left(w_{h}\right)\right\rangle_{E} n_{E},\left[\left[u_{h}\right]\right]_{E}\right)_{E}\right) .
\end{aligned}
$$

The first term is estimated in a standard way. The second term is also estimated in a classical way, namely

$$
\sum_{E \in \mathcal{E}_{h}}\left(\left[\left[\sigma_{h}\left(u_{h}\right) \cdot n_{E}\right]\right], w_{h}\right)_{E}=\sum_{E \in \mathcal{E}_{h}}\left(\left[\left[\sigma_{h}\left(u_{h}\right) \cdot n_{E}\right]\right], w_{h}-w\right)_{E},
$$

and therefore by classical local error estimates on the Clément interpolant ([29], Lemma 1.4, p. 11) using Lemma 3.4 of [11], we get

$$
\left|\sum_{E \in \mathcal{E}_{h}}\left(\left[\left[\sigma_{h}\left(u_{h}\right) \cdot n_{E}\right]\right], w_{h}\right)_{E}\right| \lesssim \eta\|w\|_{1, \Omega} .
$$


For the last term we can write (see above)

$\left(\left\langle\sigma\left(w_{h}\right)\right\rangle_{E} n_{E},\left[\left[u_{h}\right]\right]_{E}\right)_{E}=2 \mu\left(\left\langle\epsilon\left(w_{h}\right)\right\rangle_{E} n_{E},\left[\left[u_{h}\right]\right]_{E}\right)_{E}+\lambda\left(\left\langle\operatorname{div}_{h} w_{h}\right\rangle_{E},\left[\left[P_{0} u_{h} \cdot n_{E}\right]\right]_{E}\right)_{E}$.

Hence using scaling arguments, we obtain

$$
\left|\sum_{E \in \mathcal{E}_{h}}\left(\left\langle\sigma\left(w_{h}\right)\right\rangle_{E} n_{E},\left[\left[u_{h}\right]\right]_{E}\right)_{E}\right| \lesssim\left\|w_{h}\right\|_{1, \Omega}\left(\sum_{E \in \mathcal{E}_{h}} \frac{1}{h_{E}}\left(\left\|\left[\left[u_{h}\right]\right]\right\|_{E}^{2}+\lambda^{2}\left\|\left[\left[P_{0} u_{h} \cdot n_{E}\right]\right]\right\|_{E}^{2}\right)\right)^{\frac{1}{2}} .
$$

Hence using again Lemma 1.4 of [29], we get

$$
\left|\sum_{E \in \mathcal{E}_{h}}\left(\left\langle\sigma\left(w_{h}\right)\right\rangle_{E} n_{E},\left[\left[u_{h}\right]\right]_{E}\right)_{E}\right| \lesssim \eta\|w\|_{1, \Omega} .
$$

The third term of (4.5) is estimated by using (4.3), namely

$$
\left|2 \mu\left(\epsilon\left(u_{h}^{\perp}\right), \epsilon(w)\right)\right| \lesssim\left\|\nabla_{h} u_{h}^{\perp}\right\|\|\nabla w\| \lesssim \eta\|\nabla w\| .
$$

For the last term of (4.5) since $e^{c}$ belongs to $H_{0}^{1}(\Omega)^{2}$ its divergence is of mean zero and therefore as in [11], we find

$$
-\lambda\left(\operatorname{div}_{h} e, \operatorname{div} e^{c}\right) \leq-\lambda\left(\operatorname{div}_{h} e, \operatorname{div}_{h} u_{h}^{\perp}\right) .
$$

Hence by Cauchy-Schwarz's inequality and the estimate (4.3), we again arrive at

$$
-\lambda\left(\operatorname{div}_{h} e, \operatorname{div} e^{c}\right) \lesssim \eta \lambda\left\|\operatorname{div}_{h} e\right\| \lesssim \eta\left\|\left|\|\mid\|_{\lambda} .\right.\right.
$$

All the previous estimates lead to the conclusion.

5. Lower bound of the error. For a subset $\omega$ of $\bar{\Omega}$, we denote by

$$
\begin{aligned}
\|\| \|_{\lambda, \omega}^{2} & :=\left(\sigma_{h}(e), \epsilon_{h}(e)\right)_{\omega}+\lambda^{2}\left\|\Pi_{0} \operatorname{div}_{h} e\right\|_{\omega}^{2} \\
& +\sum_{E \in \mathcal{E}_{h}: E \subset \omega} \frac{1}{h_{E}}\left(2 \mu \gamma_{1}\left\|\left[\left[u_{h}\right]\right]\right\|_{E}^{2}+\lambda^{2}\left\|\left[\left[P_{0} u_{h} \cdot n_{E}\right]\right]\right\|_{E}^{2}\right), \\
o s c_{\omega}^{2}(f) & :=\sum_{T \in \mathcal{T}_{h}: T \subset \omega} h_{T}^{2}\left\|f-f_{T}\right\|_{T}^{2} .
\end{aligned}
$$

As usual, for any triangle $T$ we denote by

$$
\omega_{T}=\cup_{T^{\prime} \in \mathcal{T}_{h}: T^{\prime} \cap T \neq \emptyset} T^{\prime},
$$

the patch associated with $T$. Similarly for an edge $E$, we set

$$
\omega_{E}=\cup_{T^{\prime} \in \mathcal{T}_{h}: E \subset T^{\prime}} T^{\prime} .
$$

We start with the element residual.

Lemma 5.1. For all $T \in \mathcal{T}_{h}$, we have

$$
h_{T}\left\|f_{T}\right\|_{T} \lesssim\|\| e \|_{\lambda, T}+\operatorname{osc}_{T}(f) .
$$


Proof. If $b_{T}$ is the standard bubble function associated with $T$, we set $w_{T}=f_{T} b_{T}$. Hence by a standard inverse inequality we have

$$
\left\|f_{T}\right\|_{T}^{2} \lesssim \int_{T} f_{T} \cdot w_{T} d x=\int_{T}\left(f_{T}-f\right) \cdot w_{T} d x-\int_{T} \operatorname{div} \sigma\left(u-u_{h}\right) \cdot w_{T} d x
$$

reminding that $\operatorname{div} \sigma(u)=-f$. For the second term of this right-hand side applying Green's formula we obtain

$$
\left\|f_{T}\right\|_{T}^{2} \lesssim \int_{T}\left(f_{T}-f\right) \cdot w_{T} d x+\int_{T} \sigma\left(u-u_{h}\right): \epsilon\left(w_{T}\right) d x .
$$

Now we can write

$$
\begin{aligned}
\int_{T} \sigma\left(u-u_{h}\right): \epsilon\left(w_{T}\right) d x & =2 \mu \int_{T} \epsilon\left(u-u_{h}\right): \epsilon\left(w_{T}\right) d x+\lambda \int_{T} \operatorname{div}\left(u-u_{h}\right) \operatorname{div}\left(w_{T}\right) d x \\
& =2 \mu \int_{T} \epsilon\left(u-u_{h}\right): \epsilon\left(w_{T}\right) d x+\lambda \int_{\Omega} \operatorname{div}_{h}\left(u-u_{h}\right) \operatorname{div}\left(w_{T}\right) d x .
\end{aligned}
$$

Since $w_{T}$ can be seen as a function in $H_{0}^{1}(\Omega)^{2}$, we deduce that

$$
\begin{aligned}
\int_{T} \sigma\left(u-u_{h}\right): \epsilon\left(w_{T}\right) d x & =2 \mu \int_{T} \epsilon\left(u-u_{h}\right): \epsilon\left(w_{T}\right) d x+\lambda \int_{\Omega} \Pi_{0} \operatorname{div}_{h}\left(u-u_{h}\right) \operatorname{div}\left(w_{T}\right) d x \\
& =2 \mu \int_{T} \epsilon\left(u-u_{h}\right): \epsilon\left(w_{T}\right) d x+\lambda \int_{T} \Pi_{0} \operatorname{div}_{h}\left(u-u_{h}\right) \operatorname{div}\left(w_{T}\right) d x .
\end{aligned}
$$

By Cauchy-Schwarz's inequality and the inverse inequality $\left\|\epsilon\left(w_{T}\right)\right\|_{T} \lesssim h_{T}^{-1}\left\|f_{T}\right\|_{T}$, we obtain

$$
h_{T}\left\|f_{T}\right\|_{T} \lesssim \operatorname{osc}_{T}(f)+\left\|\epsilon\left(u-u_{h}\right)\right\|_{T}+\lambda\left\|\Pi_{0} \operatorname{div}_{h}\left(u-u_{h}\right)\right\|_{T} .
$$

The conclusion follows from the direct estimate

$$
\|\epsilon(e)\|_{T}^{2} \leq \frac{1}{2 \mu} \int_{T} \sigma(e): \epsilon(e) d x
$$

We argue in a similar manner for the normal jump, namely we have the Lemma 5.2. For all $E \in \mathcal{E}_{h}$, we have

$$
h_{E}^{1 / 2}\left\|\left[\left[\sigma\left(u_{h}\right) \cdot n_{E}\right]\right]_{E}\right\|_{E} \lesssim\||e|\|_{\lambda, \omega_{E}}+o s c_{\omega_{E}}(f) .
$$

Proof. Denoting by $b_{E}$ the edge bubble we set $w_{E}=\left[\left[\sigma\left(u_{h}\right) \cdot n_{E}\right]\right]_{E} b_{E}$ that belongs to $\left[H_{0}^{1}\left(\omega_{E}\right)\right]^{2}$. Hence by a standard inverse inequality we have

$$
\begin{aligned}
\left\|\left[\left[\sigma\left(u_{h}\right) \cdot n_{E}\right]\right]_{E}\right\|_{E}^{2} & \sim \int_{E}\left[\left[\sigma\left(u_{h}\right) \cdot n_{E}\right]\right]_{E} \cdot w_{E} d s=\int_{E}\left[\left[\sigma\left(u_{h}-u\right) \cdot n_{E}\right]\right]_{E} \cdot w_{E} d s \\
& =\sum_{T \in \mathcal{T}_{h}: T \subset \omega_{E}} \int_{\partial T} \sigma\left(u_{h}-u\right) \cdot n \cdot w_{E} d s .
\end{aligned}
$$

Hence an integration by parts yields

$$
\left\|\left[\left[\sigma\left(u_{h}\right) \cdot n_{E}\right]\right]_{E}\right\|_{E}^{2} \sim \sum_{T \in \mathcal{T}_{h}: T \subset \omega_{E}}\left(\int_{T} \sigma\left(u_{h}-u\right): \nabla\left(w_{E}\right) d x+\int_{T} f \cdot w_{E} d x\right) .
$$


Using the same argument as before and Cauchy-Schwarz's inequality and inverse inequalities we obtain

$$
\begin{aligned}
h_{E}^{1 / 2}\left\|\left[\left[\sigma\left(u_{h}\right) \cdot n_{E}\right]\right]_{E}\right\|_{E} & \lesssim \sum_{\substack{T \in \mathcal{T}_{h}: T \subset \omega_{E}\\
}}\left(\left\|\epsilon\left(u_{h}-u\right)\right\|_{T}+h_{T}\left\|f_{T}\right\|_{T}\right) \\
& +\lambda\left\|\Pi_{0} \operatorname{div}_{h}\left(u-u_{h}\right)\right\|_{\omega_{E}}+o s c_{\omega_{E}}(f) .
\end{aligned}
$$

The conclusion follows from Lemma 5.1 and the estimate (5.1).

These two lemmas directly yield the

Theorem 5.3. For all $T \in \mathcal{T}_{h}$, we have

$$
\eta_{T} \lesssim|||e| \|_{\lambda, \omega_{T}}+o s c_{\omega_{T}}(f) .
$$

6. The nonconforming Galerkin method based on the Crouzeix-Raviart element. The arguments of the three previous section can be directly applied to the discontinuous method (2.10). They are even simpler because $\left[\left[P_{0} u_{h} \cdot n\right]\right]_{E}=0$ on any edge $E$. Hence we have not to use the extra term

$$
\lambda^{2} \sum_{E \in \mathcal{E}_{h}} \frac{1}{h_{E}}\left\|\left[\left[P_{0} e \cdot n\right]\right]\right\|_{E}^{2} .
$$

in the norm of the error (because it is zero).

More precisely we can prove the next results:

THEOREM 6.1. We have the following a posteriori estimate:

$$
\||| e\|+\lambda\left\|\Pi_{0} \operatorname{div}_{h} e\right\| \lesssim \eta_{0}+o s c(f),
$$

where

$$
\eta_{0}^{2}=\sum_{T \in \mathcal{T}_{h}} \eta_{0 T}^{2}
$$

and for all $T \in \mathcal{T}_{h}$, the local estimator $\eta_{0 T}$ is defined by

$$
\eta_{0 T}^{2}=h_{T}^{2}\left\|f_{T}\right\|_{T}^{2}+\sum_{E \in \mathcal{E}_{h}: E \subset T} h_{E}\left\|\left[\left[\sigma\left(u_{h}\right) \cdot n_{E}\right]\right]_{E}\right\|_{E}^{2}+\sum_{E \in \mathcal{E}_{h}: E \subset T} \frac{1}{h_{E}}\left\|\left[\left[u_{h}\right]\right]\right\|_{E}^{2} .
$$

Theorem 6.2. For all $T \in \mathcal{T}_{h}$, we have

$$
\eta_{0 T} \lesssim\||| e \mid\|_{\omega_{T}}+\lambda\left\|\Pi_{0} \operatorname{div}_{h}(e)\right\|_{\omega_{T}}+o s c_{\omega_{T}}(f),
$$

where

$$
\|\|\left\|_{\omega}^{2}:=\sum_{T \in \mathcal{T}_{h}: T \subset \omega}(\sigma(e), \epsilon(e))_{T}+2 \mu \gamma_{1} \sum_{E \in \mathcal{E}_{h}: E \subset \omega} \frac{1}{h_{E}}\right\|\left[\left[u_{h}\right]\right] \|_{E}^{2} .
$$

Theorem 6.1 is also a consequence of Theorem 2.1 of [15] since $u_{0 h}$ satisfies the assumptions of this theorem. We give here an alternative proof.

7. Numerical experiments. In this section, some numerical experiments are performed to illustrate the above theoretical results, similarly to the ones proposed in [11]. In all the following cases, Crouzeix-Raviart finite elements $C R\left(\mathcal{T}_{h}\right)$ defined by (2.5) are used on a regular mesh composed of triangles. 
7.1. Divergence-free smooth solutions. The first test consists in solving equation $(2.1)$ on the unit square $\Omega=(0,1)^{2}$, for the exact solution given by :

$$
u(x, y)=\frac{1}{\pi^{2}}(\sin (\pi x) \sin (\pi y))^{n-1}\left(\begin{array}{c}
\sin (\pi x) \cos (\pi y) \\
-\sin (\pi y) \cos (\pi x)
\end{array}\right),
$$

where $n \geq 2$ is a given integer and where $f$ is chosen accordingly. It should be noted that $u$ is smooth, divergence-free, and that $u_{\mid \partial \Omega}=0$.

For the first example, we set $n=2$. We plot in Figure 7.1 the convergence rates of the error (Figure 7.1 (a)) and of the estimator (Figure 7.1 (b)) for several values of $\lambda$, using a uniform mesh refinement strategy. It can be observed a good convergence towards zero for both of them corresponding to the one theoretically expected (namely, order 1 in $h$ corresponding to order $-1 / 2$ in the total number of degrees of freedom $n d o f)$.

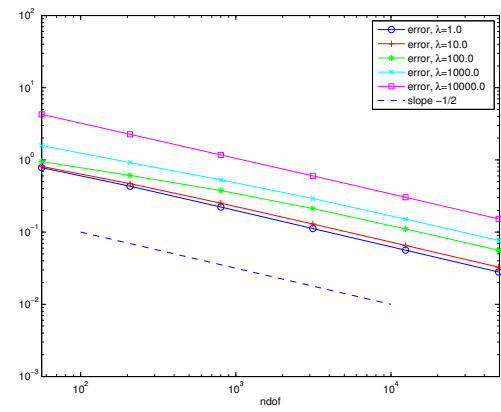

(a)

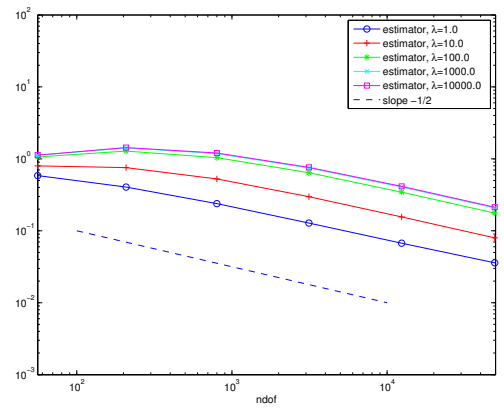

(b)

FIG. 7.1. Error (a) and Estimator (b) convergence rates in ndof for the regular solution, $n=2$, $\lambda$ from 1 to $10^{4}$.

Then, the effectivity index defined by :

$$
I_{e f f}=\frac{\eta_{0}}{\left\|\left|\|\mid\|+\lambda\left\|\Pi_{0} \operatorname{div}_{h} e\right\|\right.\right.}
$$

is plotted in Figure 7.2. First, it can be observed that for a given value of $\lambda$, the reliability of the estimator is ensured, as underlined by Theorem 6.1. Moreover, even if the value of $I_{\text {eff }}$ is not totally independant of $\lambda$, it remains in a reasonable range (in the order of 1 ).

For the second example, we set $n=25$ in order to generate high gradients in the vicinity of the mid-point $\left(\frac{1}{2}, \frac{1}{2}\right)$. Consequently, instead of a uniform one, a local refinement mesh strategy is performed based on the local error estimator efficiency proven in Theorem 6.2. Here, this local refinement ensures that the mesh remains conform (there is no hanging-nodes creation), as well as regular (the minimal angle allowed in the triangulation is specified, in order to preserve the shape regularity of the mesh). We refer to [29] for further details and for the algorithm description (red, green and blue refinement). The resulting meshes obtained for iteration steps 1, 4 and 7 corresponding to $\lambda=10^{4}$ are displayed in Figure 7.3, so that we can observe that the high gradients area is well tracked by the remeshing process. The convergence rates 


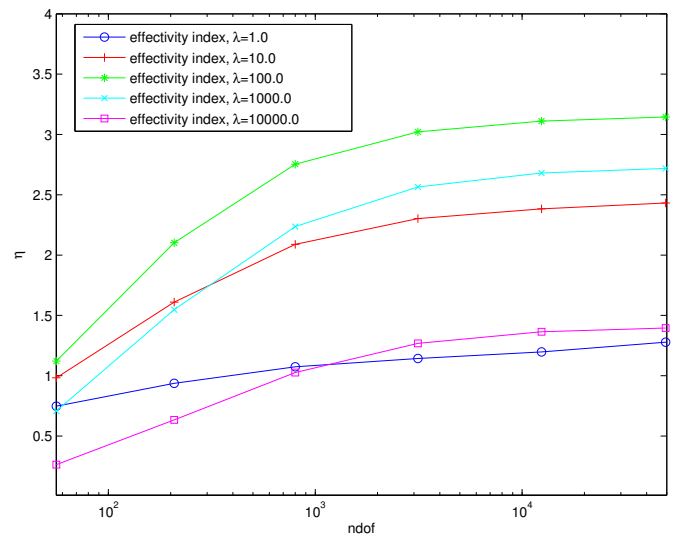

FIG. 7.2. Effectivity index $I_{\text {eff }}$ for the regular solution, $n=2, \lambda$ from 1 to $10^{4}$.

of the error and of the estimator are plotted in Figure 7.4 and the effectivity index in Figure 7.5. Once again, the conclusions are the same than for the first example: Experimental convergence rates of order 1 and robustness with respect to large values of $\lambda$, as well as only a small variation in $\lambda$ of the effectivity index (around 1) for all the considered values of $\lambda$.
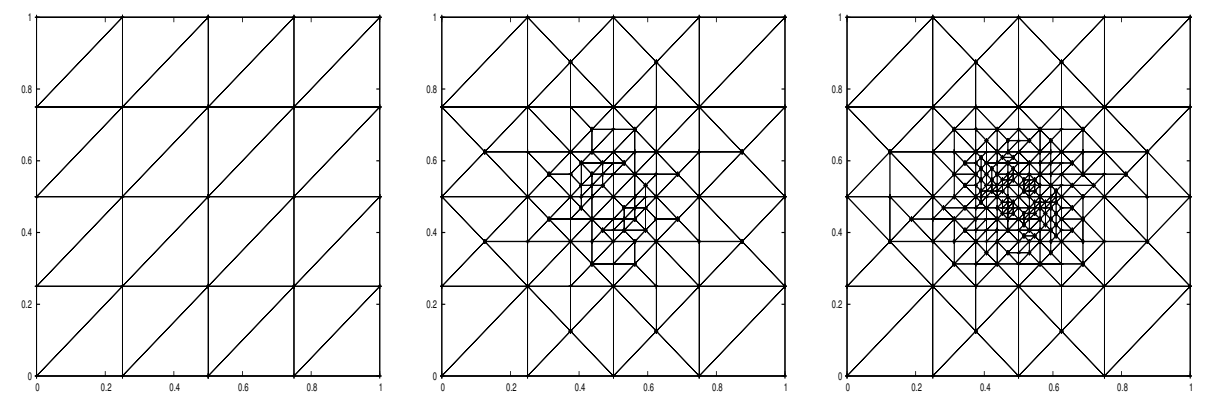

FIG. 7.3. Mesh refinements 1,4 and 7 for the regular solution, $n=25, \lambda=10^{4}$.

7.2. Singular solution. This second test consists now in solving equation (2.1) on the domain $\Omega$ defined by :

$$
\Omega=(-1,1)^{2} \backslash\{(x, y) ;-0.1 x \leq y \leq 0.1 x, 0 \leq x\},
$$

with the external force $f$ chosen to be :

$$
f(x, y)=-r^{-\frac{3}{4}}\left(\begin{array}{l}
1 \\
0
\end{array}\right), \quad r=\sqrt{x^{2}+y^{2}} .
$$

In that case, the exact solution exhibits a singular behaviour around the origin. We want to check if the mesh is correctly refined around the singularity when the estimator is used to carry out the refinement process, and in the same time if the global estimator decreases consequently towards zero. The resulting meshes obtained for iteration steps 


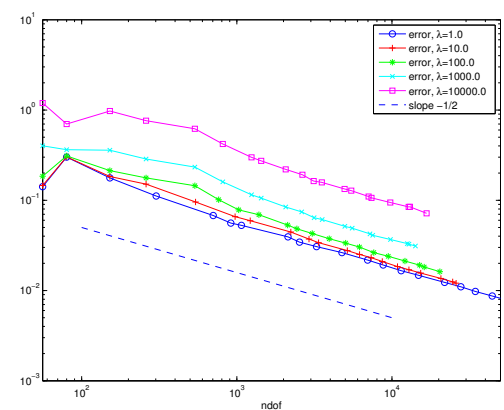

(a)

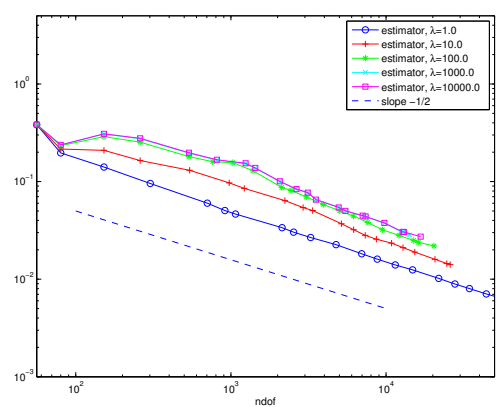

(b)

FIG. 7.4. Error (a) and Estimator (b) convergence rates in ndof for the regular solution, $n=25, \lambda$ from 1 to $10^{4}$.

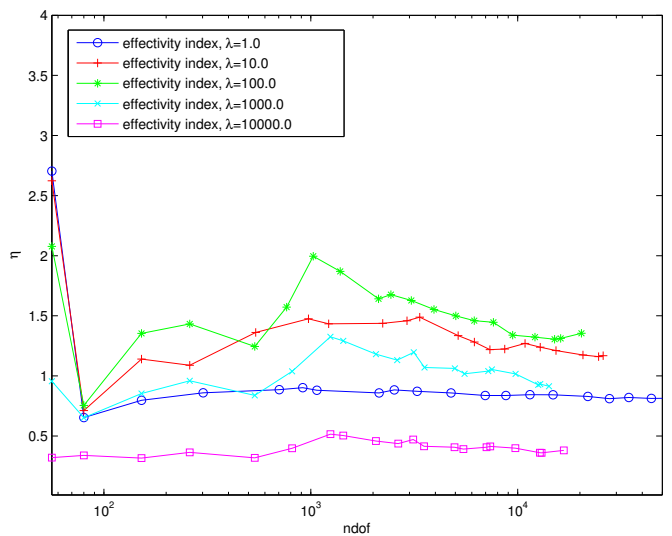

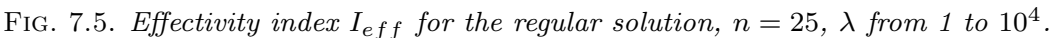

1, 7 and 10 corresponding to $\lambda=10^{4}$ are displayed in Figure 7.6, and we can see that the mesh is automatically refined around the singularity. Moreover, Figure 7.7 shows that the estimator converges towards zero, with experimental convergence rates of order 1. 

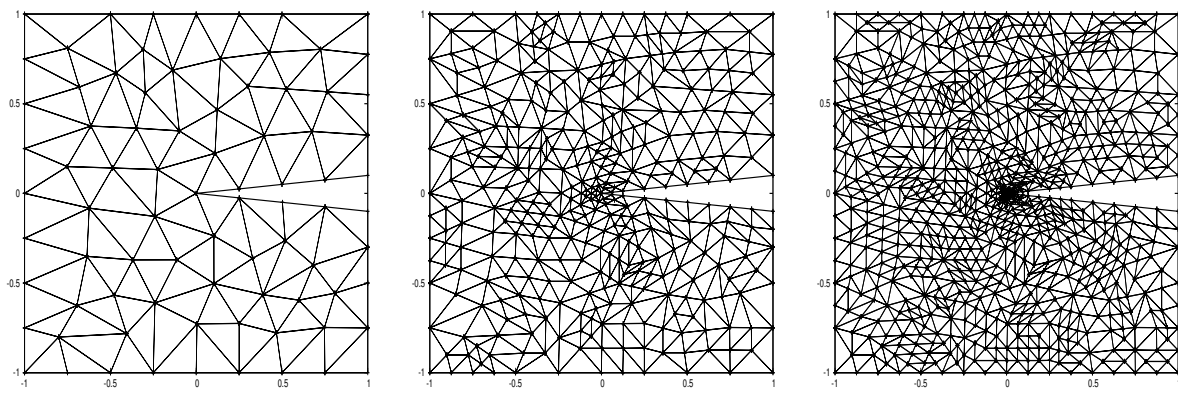

FIG. 7.6. Mesh refinements 1,7 and 10 for the singular solution, $\lambda=10^{4}$.

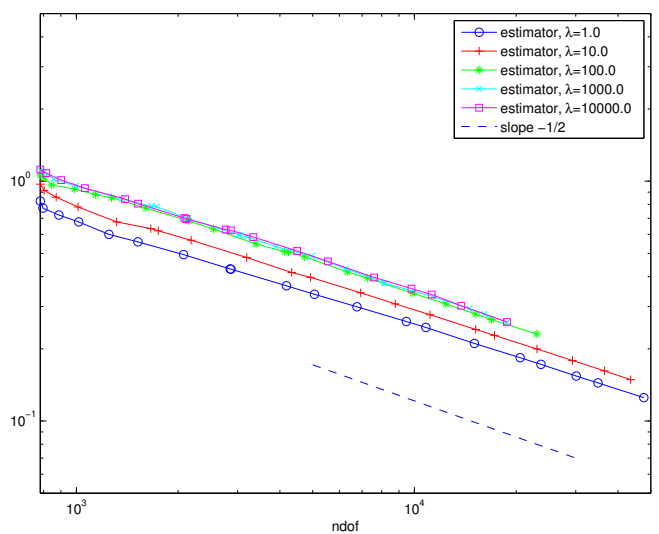

FIG. 7.7. Estimator convergence rate in ndof for the singular solution, $\lambda$ from 1 to $10^{4}$. 


\section{REFERENCES}

[1] M. Ainsworth And I. BABUŠKa, Reliable and robust a posteriori error estimation for singularly perturbed reaction-diffusion problems, SIAM J. Numer. Anal., 36 (1998), pp. 331-353.

[2] M. Ainsworth And J. Oden, A posteriori error estimation in finite element analysis, Chichester: Wiley, 2000.

[3] M. Ainsworth and J. T. Oden, A posteriori error estimators for 2nd order elliptic systems II. an optimal order process for calculating self-equilibrated fluxes, Comput. Math. Appl., 26 (1993), pp. $75-87$.

[4] I. BabušKa, R. Duran, And R. Rodriguez, Analysis of the efficiency of an a posteriori error estimator for linear triangular elements, SIAM J. Numer. Anal., 29 (1992), pp. 947-964.

[5] I. BABUŠKa AND A. Miller, A feedback finite element method with a posteriori error estimation. I: The finite element method and some basic properties of the a posteriori error estimator, Comput. Methods Appl. Mech. Eng., 61 (1987), pp. 1-40.

[6] I. BABUŠKa And W. Rheinboldt, A posteriori error estimates for the finite element method, Int. J. Numer. Methods Eng., 12 (1978), pp. 1597-1615.

[7] I. BabušKa And T. Strouboulis, The finite element methods and its reliability, Oxford: Clarendon Press, 2001.

[8] R. E. Bank And A. Weiser, Some a posteriori error estimators for elliptic partial differential equations, Math. Comp., 44 (1985), pp. 283-301.

[9] L. Boulahjine, M. Farhloul, and L. Paquet, A posteriori error estimation for the dual mixed finite element method of the elasticity problem in a polygonal domain, Numer. Methods Partial Differential Equations, 21 (2005), pp. 938-960.

[10] S. C. Brenner And L. R. Scott, The Mathematical Theory of Finite Element Methods, Springer Verlag, New York, 1994.

[11] L. J. BRIDgeman And T. P. Wihler, Stability and a posteriori error analysis of discontinuous Galerkin methods for linearized elasticity, Comput. Methods Appl. Mech. Engrg., 200 (2011), pp. 1543-1557.

[12] U. BRInk AND E. Stein, A posteriori error estimation in large-strain elasticity using equilibrated local Neumann problems, Comput. Methods Appl. Mech. Eng., 161 (1998), pp. 77101.

[13] C. Carstensen and G. Dolzmann, A posteriori error estimates for mixed FEM in elasticity, Numer. Math., 81 (1998), pp. 187-209.

[14] C. Carstensen, G. Dolzmann, S. A. Funken, and D. S. Helm, Locking-free adaptive mixed finite element methods in linear elasticity, Comput. Methods Appl. Mech. Engrg., 190 (2000), pp. 1701-1718.

[15] C. Carstensen and S. A. Funken, Averaging technique for a posteriori error control in elasticity. III. Locking-free nonconforming FEM, Comput. Methods Appl. Mech. Engrg., 191 (2001), pp. 861-877.

[16] — - A posteriori error control in low-order finite element discretisations of incompressible stationary flow problems, Math. Comp., 70 (2001), pp. 1353-1381 (electronic).

[17] C. Carstensen and J. Hu, A unifying theory of a posteriori error control for nonconforming finite element methods, Numer. Math., 107 (2007), pp. 473-502.

[18] P. G. Ciarlet, The finite element method for elliptic problems, North-Holland, Amsterdam, 1978.

[19] F. Frey, Analyse des Structures et Milieux Continus Mécanique des Solides, vol. 3 of Traité de Génie Civil de l'EPFL, Presses Polytechniques et Universitaires Romandes, 1998.

[20] P. Hansbo and M. G. Larson, Discontinuous Galerkin and the Crouzeix-Raviart element: application to elasticity, M2AN Math. Model. Numer. Anal., 37 (2003), pp. 63-72.

[21] P. Houston, I. Perugia, and D. Schötzau, Mixed discontinuous Galerkin approximation of the Maxwell operator: Non-stabilized formulation, J. Scientific Computing, 22 (2005), pp. 315-346.

[22] P. Houston, D. Schötzau, and T. P. Wihler, An hp-adaptive mixed discontinuous Galerkin FEM for nearly incompressible linear elasticity, Comput. Methods Appl. Mech. Engrg., 195 (2006), pp. 3224-3246.

[23] P. Ladevèze, G. Coffignal, and J. P. Pelle, Accuracy of elastoplatic and dynamic analysis, Applied Numerical Mathematics, John Wiley \& Sons, 1986.

[24] P. Ladevèze And D. Leguillon, Error estimate procedure in the finite element method and applications, SIAM J. Numer. Anal., 20 (1983), pp. 485-509.

[25] P. Ladevìze, J. P. Pelle, and P. Rougeot, Error estimates and mesh optimization for finite element computation, Engrg. Comp., 8 (1991), pp. 69-80.

[26] S. NicAise, About the Lamé system in a polygonal or a polyhedral domain and a coupled 
problem between the Lamé system and the plate equation. I. Regularity of the solutions, Ann. Scuola Norm. Sup. Pisa Cl. Sci. (4), 19 (1992), pp. 327-361.

[27] S. Nicaise, K. Witowski, and B. I. Wohlmuth, An a posteriori error estimator for the Lamé equation based on equilibrated fluxes, IMA J. Numer. Anal., 28 (2008), pp. 331-353.

[28] V. Z. Parton and P. I. Perlin, Mathematical methods of the theory of elasticity. Vol. 1, Mir, Moscow, 1984. Translated from the Russian by Ram S. Wadhwa.

[29] R. Verfürth, A review of a posteriori error estimation and adaptive mesh-refinement techniques, Wiley-Teubner Series Advances in Numerical Mathematics, Wiley-Teubner, Chichester, Stuttgart, 1996.

[30] - A review of a posteriori error estimation techniques for elasticity problems, Comput. Methods Appl. Mech. Engrg., 176 (1999), pp. 419-440.

[31] T. P. WIHLER, Locking-free adaptive discontinuous Galerkin FEM for linear elasticity problems, Math. Comp., 75 (2006), pp. 1087-1102 (electronic).

[32] J. Z. ZhU And O. C. Zienkiewicz, Adaptive techniques in the finite element method, Commun. Appl. Numer. Methods, 4 (1988), pp. 197-204.

[33] O. C. ZIENKIEWICZ AND J. Z. ZHU, A simple error estimator and adaptive procedure for practical engineering analysis., Internat. J. Numer. Methods Engrg., 24 (1987), pp. 337-357. 